\title{
Shift work and cancer
}

\section{SLEEP DURATION AND THE RISK OF CANCER IN THE PROSPECTIVE MILLION WOMEN STUDY}

Ruth Travis, Benjamin Cairns, Xiao Si Wang, Gillian Reeves, Valerie Beral, on behalf of the Million Women Study Collaborators University of Oxford, Oxford, UK

10.1136/oemed-2011-100382.51

Objectives The International Agency for Research on Cancer has classified shift work involving circadian disruption as a probable carcinogen, promoting interest in the relationship between sleep duration and cancer risk in the general population. However, the published epidemiological data are inconclusive with respect to the association with risk for breast cancer and are scarce for cancer at other sites. The objective was to investigate the relationship between 24 -h sleep duration and the incidence of common cancers in middle-aged women. Methods In this prospective study 737143 women aged 52-72 years old provided information in 2004 on how long they slept for on average during $24 \mathrm{~h}$ and other information relevant for cancer incidence. All were followed by record linkage to cancer registration data. Cox regression models were used to calculate relative risks for all cancers, common specific cancer sites, and other unspecified cancers, according to duration of sleep at night, adjusting for a range of potential confounders.

Results and conclusions We will present findings from this prospective investigation of sleep duration in relation to breast cancer risk in a large UK cohort. 